University for Business and Technology in Kosovo

UBT Knowledge Center

UBT International Conference

2017 UBT International Conference

Oct 28th, 11:00 AM - 12:30 PM

\title{
Prevalence of Terminal Renal Disease in Lupus Nephritis Patients
}

\author{
Marsida Duli \\ Universiteti Europian i Tiranes \\ Qamil Dika \\ Universiteti Ismail Qemali, qamil_dika@yahoo.com \\ Xhenila Duli \\ Mjeke e Pergjithshme \\ Joana Hankollari \\ Albanian University, joanahankollari@gmail.com
}

Follow this and additional works at: https://knowledgecenter.ubt-uni.net/conference

Part of the Medicine and Health Sciences Commons

\section{Recommended Citation}

Duli, Marsida; Dika, Qamil; Duli, Xhenila; and Hankollari, Joana, "Prevalence of Terminal Renal Disease in Lupus Nephritis Patients" (2017). UBT International Conference. 289.

https://knowledgecenter.ubt-uni.net/conference/2017/all-events/289

This Event is brought to you for free and open access by the Publication and Journals at UBT Knowledge Center. It has been accepted for inclusion in UBT International Conference by an authorized administrator of UBT Knowledge Center. For more information, please contact knowledge.center@ubt-uni.net. 


\title{
Prevalence of Terminal Renal Disease in Lupus Nephritis Patients
}

\author{
Marsida Duli ${ }^{1}$, Joana Hankollari ${ }^{2}$, Qamil Dika ${ }^{3}$, Xhenila Duli ${ }^{4}$, Indrit Bimi ${ }^{5}$, Daniela \\ Dervishi $^{5}$ \\ ${ }^{1}$ Universiteti Europian i Tiranës \\ ${ }^{2}$ Fakulteti i Shkencave Mjekësore, Albanian University, Tiranë \\ ${ }^{3}$ Universiteti Ismail Qemali \\ ${ }^{4}$ Mjeke e përgjithshme \\ ${ }^{5}$ Alexander Moisiu University of Durrës \\ marsiduli@hotmail.com
}

\begin{abstract}
Systemic erythematosus lupus is a chronic inflammatory disease of autoimmune nature. This illness can affect all organs, but one of the most common illnesses is lupus nephritis. Patients with renal impairment, despite aggressive immunosuppressive medication carry the risk of developing terminal renal disease and doing dialysis or renal transplant. The purpose of this study is to reflect global statistics on the frequency of the development of terminal renal disease in lupus nephritis patients as an awareness-raising way to stimulate studies that present a successful treatment or screening protocol in preventing this complication. This study is a review based on the research of world studies and literature regarding the prevalence of terminal renal disease in lupus nephritis patients. Over $50 \%$ of patients with LES, depending on different regions of the world, race / ethnicity develop lupus nephritis. 10-30\% of lupus nephritis cases develop terminal renal disease where among the major risk factors are new age, nephrotic proteinuria, disease activity index, histological class of renal impairment, high creatinine, lack of remission, etc. Performing as early as possible the renal transplant increases the lifespan of patients with terminal renal disease. It is very difficult to prevent renal impairment in patients with LES, more so when some of them have lupus nephritis as the initial manifestation. However, since more than $50 \%$ of LES patients develop lupus nephritis and 10 to $30 \%$ of lupus nephritis cases develop terminal renal disease, studies should be encouraged to determine a successful treatment or screening protocol in preventing this complication.
\end{abstract}

Keywords: systemic lupus erythematosus, prevalence, lupus nephritis, terminal renal disease

\section{Introduction}

Systemic erythematosus lupus is a chronic inflammatory autoimmune disease of connective tissue. This pathology can affect every organ, but one of the most commonly affection that increases the risk of mortality is lupus nephritis. Lupus nephritis must be suspected in all cases that the patient presents the urine analysis with microscopic hematuria, proteinuria, and if the latter are associated with decreased glomerular filtration function then the patient with lupus should undergo renal biopsy. Renal biopsy is a practice that does not apply in our country. And even in developed countries with a consolidated health system, renal biopsy should be performed in specialized centers for the interpretation of its results, especially for lupus nephritis cases. 
Based on a 2003 classification, the International Society of Nephrology and the Renal Pathology Society conducted a classification of lupine nephritis. ${ }^{4}$

Class I: Minimal mesangial lupus nephritis

Class II: Mesangial proliferative lupus nephritis

Class III: Focal lupus nephritis (Affected Less than 50\% of Glomeruli)

Class III-A: Active Lesions

Class III-A / C: Active and Chronic Lesions

Class III-C: Inactive Chronic Lesions

Class IV: Diffuse lupus nephritis that can be segmental or global

Class IV-A: Active Lesions

Class IV-A / C: Active and Chronic Lesions

Class IV-C: Inactive Chronic Lesions

Class V: Membranous lupus nephritis

Class VI: Advanced sclerosing lupus nephritis.

All the cases with inactive chronic lesions have a bad prognosis.

Despite the aggressive immunosuppressive therapy, lupus nephritis can advance towards chronic terminal renal disease. The patient in this case becomes subject to dialysis, whether it is hemodialysis or peritoneal dialysis. Where possible, renal transplantation is performed. The latter is considered as the most appropriate solution to increase the life expectancy and quality of life of these patients. This purely theoretical nature study, based on world literature research, aims at reflecting the prevalence of terminal renal disease in patients affected by lupus nephritis in order to promote the development of prophylactic protocols of this complication.

\section{Early screening of lupus nephritis}

\section{Diagnosis of systemic lupus erythematosus}

It is necessary to have the diagnosis of systemic lupus erythematosus as soon as possible in order to prevent through its treatment the possible complications, which affect vital organs and increase the risk of mortality in patients. First, whether the general practitioner or the specialist with whom the patient is in contact, must be familiar with the symptoms and signs of this pathology. Early suspicion of this pathology allows the patient to be referred to the rheumatologist as soon as possible and to initiate the appropriate treatment as soon as possible. Some of the signs that the patient may display are: malar rash, photosensibilisation, alopexy, oral ulcers, vasculitis, presence of Raynaud's phenomenon, serositis, generalized articular pain, livedo reticularis etc. The rheumatologist performs appropriate objective, laboratory and imaging tests to diagnose lupus. The systemic erythematous lupus diagnosis criteria set by the American College of Rheumatology were reviewed in 2011 by Systemic Lupus International Collaborating Clinics. The revision has increased the role of immunological examinations in establishing the diagnosis such as complement levels in lupus that are expected to be reduced, and direct Coombs test's. The following table presents the diagnosis criteria. We can say that the patient suffers from this pathology if it meets four of the criteria listed in the table, including at least one clinical and one immunological criterion. The lupus is also diagnosed in a patient with the presence of positive ANA and anti ds Dna tests, and with lupus nephritis verified by renal biopsy.

\footnotetext{
${ }^{4}$ Oxford American Handbook of Rheumatology, second edition, page 336.
} 
Table 1. Diagnostic Criteria for Systemic Lupus Erythematosus by Systemic Lupus International Collaborating Clinics in 2012.

\author{
Clinical criteria \\ Acute Cutaneous Lupus \\ Chronic Cutaneous Lupus \\ Oral / nasal ulcers \\ Non-scarring alopecia \\ Arthritis \\ Serositis \\ Proteinuria (greater than $500 \mathrm{mg} / 24 \mathrm{~h}$ ) or hematuria \\ Neurological disorders \\ Hemolytic anemia \\ Leukopenia $(<4000 / \mathrm{mm} 3)$ or lymphopenia $(<1000 / \mathrm{mm} 3)$ \\ Thrombocytopenia $(<100,000 / \mathrm{mm} 3)$ \\ Immunological criteria \\ ANA \\ Anti ds DNA \\ Anti-Sm. Antibodies \\ Antiphospholipid antibodies \\ Low Complex (C3, C4 or CH50) \\ Coombs' direct test (in the absence of hemolytic anemia)
}

Neurological disorders include convulsions, psychosis, multiple mononeuritis, myelitis, cranial and peripheral neuropathy, cerebral palsy.

Evidence of antiphospholipid antibodies includes lupus anticoagulant, false positivity test for syphilis, anti-cardiolipin antibodies, mid-high titer of anti- $\beta 2$ glycoprotein antibodies.

\title{
Lupus nephritis
}

Systemic erythematosus lupus affects the kidneys in over $50 \%$ of patients, although these percentages range from region to region, according to ethnic groups, sex, race. Lupus nephritis causes an increase in morbidity and development of end-stage renal disease. For this reason it is very important to prevent it and not just to treat lupus. Of course that is not possible in all cases, since lupus nephritis may be the first manifestation of lupus. The physician should include a complete urinary analysis in the protocol for examinations. The patient may present microscopic hematuria, albumin trace, the appearance of other cells present in the urine. At this point it is good to go into examinations by looking for proteinuria for 24 hours, monitoring of azotemia, cretininemia, and creatinine clearance. Through this monitoring we detect complications in time and fix drug doses. Protocols recommend to perform renal biopsy, which confirms the diagnosis at a time when lupus patients may have other additional pathologies such as diabetes, arterial hypertension, toxicity from taking medicines. On the other hand, it is also possible to classify the lupus nephritis and its prognosis. There are many controversial arguments regarding the performance of this invasive procedure that has its risks. Some think that there is no change in treatment, others think that kidney biopsy helps in the dosages and type of treatment and in diagnosis confirmation. 


\section{Prevalence of terminal renal disease in lupus nephritis patients.}

There are a number of prognostic factors that predict the risk the patient has to develop end stage type renal impairment. These risks should be considered to keep the patient under strict supervision. Among the major risk factors are new age, nephrotic proteinuria, disease activity index, histological class of renal dysfunction, high creatinine clearance, lack of remission, etc. Over $50 \%$ of systemic lupus erythematosus patients develop lupus nephritis and about 10 to $30 \%$ of lupus nephritis patients develop end-stage renal disease. The worst prognosis is in cases of chronic inactive lesions, ie glomerulosclerosis, and those cases which, despite aggressive therapy, fail to remedy the disease. Serious cases are also the patients who have like first manifestation the lupus nephritis. The co-existence of antiphospholipid antibodies complicates more the situation. The following table shows the prevalence of clinical manifestations in lupus nephritis patients.

Table 2. Prevalence of clinical manifestations in lupus nephritis patients. ${ }^{5}$

\begin{tabular}{|l|c|}
\hline Clinical manifestations & Prevalence in percentage \% \\
\hline Proteinuria & $100 \%$ \\
Nephrotic syndrome & $50 \%$ \\
Microscopic hematuria & $80 \%$ \\
Macroscopic hematuria & $<5 \%$ \\
Presence of other cell in the urine & $30 \%$ \\
Renal failure & $60 \%$ \\
Decreased renal function & $15 \%$ \\
Hypertension & $30 \%$ \\
Tubular anormalities & $70 \%$ \\
\hline
\end{tabular}

As we see from the table, in all cases with lupine nephritis there is proteinuria, then in $80 \%$ of cases microscopic hematuria, in $60 \%$ of cases the situation is complicated with renal failure, $50 \%$ nephrotic syndrome, tubular abnormalities in $70 \%$ of cases .

Some of the problems with lupus and end-stage kidney disease included discussions such as:

1. Should transplants be performed or is dialysis more effective in these patients?

2. Which dialysis would be most advisable?

3. Other Risks These Patients Have.

It has been observed that patients who undergo renal transplant have a life expectancy and better life quality than the patients who undergo dialysis. The patient should be included in the waiting list for transplantation in the early stages of the occurrence of this complication, and the lupus should be inactive. Because of the waiting time, not finding a transplant organ, economic opportunities, patients are most likely to undergo dialysis. Both forms of dialysis, whether peritoneal, or hemodialysis, have their own risks and benefits. Peritoneal dialysis carries the risk

5 Salem Almaani, Alexa Meara, Brad H.Rovin, Update on Lupus Nephritis, American Society of Nephrology, CJASN ePress, published on November 7,2016. doi: 10.2215/CJN.05780616. 
of developing peritonitis, while hemodialysis carries the risk of antiphospholipid antibody formation, which then affects the performance of the transplanted organ, as it may cause vascular thrombosis or vascular stenosis. Not only the exposure to dialysis, but also the use of aggressive immunosuppressive and glucocorticoid therapy causes these patients to have high risk of developing severe life-threatening infections. An early diagnosis of lupus is required, early diagnosis of renal complication, to further prevent terminal renal disease.

\section{Conclusions}

It is very difficult to prevent renal complications in patients with LES, more so when some of them have lupus nephritis as the initial manifestation. However, since more than 50\% of LES patients develop lupus nephritis and 10 to $30 \%$ of the latter develop terminal renal disease, studies should be encouraged to determine a successful treatment or screening protocol in preventing this complication. It has been observed that patients who undergo renal transplant have a life expectancy and better life quality than the patients who undergo dialysis. The patient should be included in the waiting list for transplantation in the early stages of the occurrence of this complication, and the lupus should be inactive. Because of the waiting time, not finding a transplant organ, economic opportunities, patients are most likely to undergo dialysis.

\section{References}

1. Salem Almaani, Alexa Meara, Brad H.Rovin, Update on Lupus Nephritis, American Society of Nephrology, CJASN ePress, published on November 7,2016. doi: 10.2215/CJN.05780616.

2. David Cucchiari, Giorgio Graziani, Claudio Ponticelli, The dialysis scenario in patients with systemic lupus erythematosus, Nephrology Dialysis Transplantation, Volume 29, Issue 8, 1 August 2014, Pages 1507-1513

3. Karen H. Costenbader, Amrita Desai, Graciela S. Alarcon, Linda Hiraki, Tamara Shaykevich, M.Alan Brookhart, Elena Massarotti, Bing Lu, Daniel H. Solomon, Wolfgang C.Winkelmayer, Trends in the Incidence, Demographics and Outcomes of End-Stage Renal Disease due to Lupus Nephritis in the U.S., 1995-2006, NIH public access, Arthritis Rheum.2011 june; 63 (6): 1681-1688. Doi: 10.1008/art.30293.

4. Pons-Estel Gj, Alarcon GS, Scofield L, Reinlib L, Cooper GS: Understanding the epidemiology and progression of systemic lupus erythematosus. Semin Arthritis Rheum 39: 257-268, 2010.

5. Huong DL, Papo T, Beaufils H, et al. Renal involvement in systemic lupus erythematosus. A study of 180 patients from a single center. Medicine ( Baltimore). 1999; 78:148-66. [ Pub.Med: 10352647].

6. Ward MM. Changes in the incidence of endstage renal disease due to lupus nephritis in the United States, 1996-2004, J Rheumatol 2009, 36: 63-67.

7. Adler M, Chambers S, Edwards C, Neild G, Isenberg D. An assessment of renal failure in an SLE cohort with special reference to ethnicity, over a 25-year period. Rheumatology (Oxford). 2006; 45: 1144-7. [ PubMed: 16527882]. 